\title{
SORL1 variants across Alzheimer's disease European American cohorts
}

\author{
Maria Victoria Fernández ${ }^{1,2}$, Kathleen Black ${ }^{1,2}$, David Carrell ${ }^{1}$, Ben Saef ${ }^{1,2}$, John Budde ${ }^{1,2}$, Yuetiva Deming ${ }^{1,2}$, \\ Bill Howells ${ }^{1,2}$, Jorge L Del-Aguila ${ }^{1,2}$, Shengmei $\mathrm{Ma}^{1,2}$, Catherine $\mathrm{Bi}^{1,2}$, Joanne Norton ${ }^{1,2}$, Rachel Chasse ${ }^{1,2}$, \\ John Morris ${ }^{2,3,4}$, Alison Goate ${ }^{5}$ and Carlos Cruchaga ${ }^{\star, 1,2}$ NIA-LOAD family study group, NCRAD
}

The accumulation of the toxic $A \boldsymbol{\beta}$ peptide in Alzheimer's disease (AD) largely relies upon an efficient recycling of amyloid precursor protein (APP). Recent genetic association studies have described rare variants in SORL1 with putative pathogenic consequences in the recycling of APP. In this work, we examine the presence of rare coding variants in SORL1 in three different European American cohorts: early-onset, late-onset AD (LOAD) and familial LOAD.

European Journal of Human Genetics (2016) 24, 1828-1830; doi:10.1038/ejhg.2016.122; published online 21 September 2016

\section{INTRODUCTION}

The main pathogenic feature of Alzheimer's disease (AD) is the accumulation of amyloid beta $(A \beta)$ peptide in brain intracellular compartments. ${ }^{1}$ Elements involved in the transport and recycling of the amyloid precursor protein (APP) have been considered targets for genetic studies to find AD risk factors. By performing association studies between genetic variants in genes of the endocytic pathway and AD, Rogaeva et al. ${ }^{2}$ first identified 19 common SNPs and two haplotypes of the neuronal sortilin-related receptor SORL1 to be associated with late-onset Alzheimer's disease (LOAD). SORL1 is a member of the retromer complex that directly binds to APP and differentially regulates its sorting into endocytic or recycling pathways. Despite some initial mixed results, ${ }^{3,4}$ recent studies provide supporting evidence for the association of SORL1 with AD risk..$^{5-8}$ Nonetheless, clear pathogenic variants that cause SORL1 malfunctioning have not yet been identified. Recently, Vardarajan et al. ${ }^{9}$ suggested that coding variants in SORL1 may be involved in LOAD pathology. They described up to 17 exonic variants significantly associated with the disease $\left(P_{\text {adjusted }}=0.008\right)$ in a family-based Caribbean-Hispanic data set. Nicolas et al. ${ }^{10}$ listed 24 rare variants in SORL1 with a cumulative effect of an odds ratio $(\mathrm{OR})=5.03\left(P=7.49 \times 10^{-5}\right)$ in a French cohort of early-onset Alzheimer's disease (EOAD) patients. However, these findings have not been replicated on independent data sets. Considering that genetic variants, and particularly rare variants, in risk factors are prone to be cohort specific, it is imperative to analyze this gene across different populations. The goal of this study was to evaluate and replicate the presence of non-synonymous variants in SORL1 gene that may increase the risk for $\mathrm{AD}$ in three different samples of the European American population: sporadic early-onset Alzheimer's disease (sEOAD), sporadic late-onset Alzheimer's disease (sLOAD) and familial LOAD (fLOAD; Table 1).

\section{MATERIALS AND METHODS}

All samples included in this analysis were recruited by the Charles F. and Joanne Knight Alzheimer's Disease Research Center (KnightADRC) and the National Institute on Aging Genetics Initiative for Late-Onset Alzheimer's Disease (NIA-LOAD). sEOAD samples came from the Memory and Aging Project (MAP), part of Washington University School of Medicine's (WUSM) Knight-Alzheimer's Disease Research Consortium (ADRC). The Institutional Review Board at the WUSM in Saint Louis approved the study. Research was carried out in accordance with the approved protocol. Written informed consent was obtained from participants and their family members by the Clinical and Genetics Core of the Knight-ADRC. The approval number for the Knight-ADRC Genetics Core family studies is 201104178.

\section{Genetic data}

sEOAD samples were genotyped for 54 variants: 24 variants reported by Nicolas et al. ${ }^{10}$ as well as 27 non-synonymous variants with $\mathrm{MAF}<5 \%$ based on the EVS database (http://evs.gs.washington.edu/ EVS/) (Supplementary Table S1), MassARRAY (Agena Biosciences) or KASP Assay (LGC Genomics, Teddington, UK).

sLOAD samples were genotyped using the Human Exome BeadChip v1.0 (Illumina, San Diego, CA, USA) technology. Stringent quality controls for exome array calling were performed. Genotype calling was carried out using Illumina's GenTrain version 1.0 clustering algorithm in GenomeStudio version 2011.1. Cluster boundaries were determined using study samples for only the calls with an intensity signal $>0.3$. A minimum call rate of $98 \%$ was used to exclude SNPs and individuals. SNPs that were not in Hardy-Weinberg equilibrium $\left(P<10^{-6}\right)$ were dropped. Pairwise genome-wide estimates of proportion identity-by-descent was used to test for unanticipated duplicates and cryptic relatedness.

fLOAD samples were sequenced using either whole-exome sequencing (WES, $n=1177$ ) or whole-genome sequencing (WGS, $n=59$ ). Exome

${ }^{1}$ Department of Psychiatry, Washington University School of Medicine, St. Louis, MO, USA; ${ }^{2}$ Hope Center for Neurological Disorders, Washington University School of Medicine, St. Louis, MO, USA; ${ }^{3}$ Knight Alzheimer's Disease Research Center, Washington University School of Medicine, St. Louis, MO, USA; ${ }^{4}$ Department of Neurology, Washington University School of Medicine, St. Louis, MO, USA; ${ }^{5}$ Department of Neuroscience, Icahn School of MEdicine at Mount Sinai, NY, USA

${ }^{*}$ Correspondence: Dr C Cruchaga, Department of Psychiatry, Washington University School of Medicine, 660 South Euclid Avenue B8134, St. Louis 63110, MO, USA. Tel: +1 314286 0546; Fax: +1 314362 2244; E-mail: ccruchaga@wustl.edu

Received 17 March 2016; revised 27 June 2016; accepted 5 August 2016; published online 21 September 2016 
Table 1 Clinical data of the three demographic groups studied

\begin{tabular}{cccccc}
\hline \multicolumn{1}{c}{$n$} & $M(\%)$ & $A A O(x \pm S D)$ & $A L A(x \pm S D)$ & $A P O E-\varepsilon 4(\%)$ \\
\hline $\begin{array}{c}S E O A D \\
\text { CO }\end{array}$ & 169 & 42.60 & & & \\
CA & 217 & 51.15 & $59.34 \pm 9.17$ & $68.93 \pm 13.68$ & 62.67 \\
& & & & & \\
SLOAD & & & & $72.51 \pm 6.24$ & 29.70 \\
CO & 266 & 41.73 & & & 28.96 \\
CA & 134 & 45.52 & $70.64 \pm 7.44$ & $75.23 \pm 6.62$ & \\
fLOAD & & & & & 51.58 \\
CO & 324 & 41.36 & & $86.57 \pm 8.25$ & 73.44 \\
CA & 866 & 35.80 & $73.34 \pm 7.42$ & $91.71 \pm 8.17$ & \\
\hline
\end{tabular}

Abbreviations: AAO, age at onset; ALA, age at last assessment; APOE- $E 4(\%)$, percentage of APOE allele 4 carriers; fLOAD, familiar late-onset Alzheimer's disease; $C A$, case; $C O$, control; $M(\%)$, percentage of males; sEOAD, sporadic early-onset Alzheimer's disease; sLOAD, sporadic late-onset Alzheimer's disease.

libraries were prepared using Agilent's SureSelect Human All Exon kits V3 and V5 (Agilent, Santa Clara, CA, USA). Both, WES and WGS samples were sequenced on a HiSeq2000 (Illumina) with paired ends reads, with a mean depth of coverage of $50-150 \times$ for WES and $30 \times$ for WGS. Variant calling was performed following GATK's 3.4 Best Practices (https://www.broadinstitute.org/gatk/). Alignment was conducted against UCSC hg19 genome reference. WES and WGS sequences were aligned and variants were called separately, following GATK's recommendations; back calling was performed to ensure that the same variants were called in both WES and WGS samples. Variant calling was restricted to Agilent's Exome capture kit with a padded 100 bp region. Only those variants and indels that fell within the above 99.9 tranche and whose quality was $\geq 30$, read depth $\geq 10$ and missingness $\leq 5 \%$; and those genotypes satisfying a genotype quality $\geq 20$ and a DP $\geq 6$ were kept for analysis. Variants with differential missingness between cases and controls, as well as between WES and WGS data sets, out of Hardy-Weinberg equilibrium $\left(P<1 \times 10^{-6}\right)$ were removed from analysis. In addition, individuals with discordant sex from that reported in the clinical database were removed from data set. Finally, individual and familial relatedness was corroborated using PLINK1.9 (https://www.cog-genomics.org/plink2/ibd) and an existing GWAS data set for these individuals. Functional annotation and population frequencies were annotated with SnpEff. ${ }^{11}$ All SORL1 variant annotations refer to sequence with Accession Number NM_003105.5. The data and phenotypes used in this study have been submitted to NIAGADS - The NIA Genetics of Alzheimer's Disease Storage Site 'https://www.niagads. org/' under accession number NG00051.

\section{Statistical analysis}

Single-variant association analysis with risk for $\mathrm{AD}$ for all data sets were performed using PLINK1.9, including significant covariates (gender and Principal Component for population stratification); for the family-based data set we used DFAM. For gene-wise analysis we only considered non-synonymous variants (missense, splice site or stop modifier) with MAF $<5 \%$ within a data set using the SNP-set Kernel Association Test (SKAT). ${ }^{12}$ The fLOAD samples were analyzed in addition via GEE Kernel Machine score test (GSKAT). ${ }^{13}$

\section{RESULTS}

\section{Sporadic EOAD}

From the 48 successfully genotyped variants in the sEOAD cohort (Supplementary Table S1), only one variant (rs117260922:G>A, hg19
Table 2 Gene-based analysis of non-synonymous and damaging SORL1 variants in each of the demographic groups studied

\begin{tabular}{|c|c|c|c|c|c|c|c|c|}
\hline \multirow[t]{2}{*}{ Test } & \multicolumn{4}{|c|}{ Non-synonymous } & \multicolumn{4}{|c|}{ PoD/PrD } \\
\hline & $n$ & $C M A F-A$ & $c M A F-U$ & P-value & $n$ & $C M A F-A$ & $c M A F-U$ & P-value \\
\hline \multicolumn{9}{|l|}{$s E O A D$} \\
\hline SKAT & 4 & 0.041 & 0.016 & 0.055 & 4 & 0.041 & 0.016 & 0.055 \\
\hline \multicolumn{9}{|l|}{$S L O A D$} \\
\hline SKAT & 17 & 0.104 & 0.111 & 0.795 & 6 & 0.052 & 0.060 & 0.945 \\
\hline \multicolumn{9}{|l|}{$f L O A D$} \\
\hline G-SKAT & 45 & & & 0.097 & 17 & & & 0.362 \\
\hline SKAT ${ }^{a}$ & 45 & & & 0.341 & 17 & & & 0.569 \\
\hline
\end{tabular}

Abbreviations: cMAF-A, cumulative minor allele frequency in affected indivdiuals; cMAF-U, cumulative minor allele frequency in unaffected individuals; fLOAD, familiar late-onset Alzheimer's disease; $\mathrm{n}$, number of SNPs included in kernel association test; PoD/PrD, possibly damaging/probably damaging according to Polyphen2; SEOAD, sporadic early-onset Alzheimer's disease; SLOAD, sporadic late-onset Alzheimer's disease.

disease; $s L O A D$, sporadic late-onset Alzheimer's disease.
aFour principal components and kinship matrix were included as covariates to account for related individuals.

chr11:g.121367627G $>$ A) was found nominally associated with $\mathrm{AD}$ status $\left(\mathrm{OR}=3.462, P_{\text {nominal }}=0.043\right)$, found in 12 cases $(n=217)$ and three controls $(n=169)$ (Supplementary Table S2). This variant was previously reported associated with LOAD risk in Hispanic families ${ }^{9}$ $\left(P=7.68 \times 10^{-7}\right)$, but we did not find a significant association in our fLOAD data set (Supplementary Table S3). Two other variants were more frequent in EOAD cases than in controls (rs140327834:T $>$ A, rs142884576:C $>$ T) although we did not find a significant difference. Nonetheless, gene-based analysis indicated there are more nonsynonymous variants in EOAD cases than in controls (collapsed MAF: $\mathrm{CA}=4.13 \% ; \mathrm{CO}=1.55 \% ; \mathrm{OR}=2.66 ; 95 \% \mathrm{CI}=0.6380-8.9850$ ) almost reaching statistically significance (SKAT $P=0.055$; Table 2).

\section{Sporadic LOAD}

From the 46 SORL1 variants genotyped in the LOAD case-control sample (134 cases and 266 controls) 16 were polymorphic (Supplementary Table S3). Four variants were present more often in cases than in controls (rs139794846:A>G, rs62617129:A $>\mathrm{G}$, rs143286467:A>G, rs143615238:G>A), although the association of any of these variants to $\mathrm{AD}$ risk was not significant in this data set $(P=0.2-0.6$; Supplementary Table S3). The gene-based analysis of the missense variants was non-significant $(\mathrm{OR}=0.992 ; P=0.979)$, and the same output was achieved when the analysis was restricted to those considered as probably or possibly damaging by Polyphen $2(\mathrm{OR}=$ 0.861, $P=0.999$; Table 2).

\section{Familial LOAD}

Within the fLOAD data set ( 875 cases and 328 controls), we identified 78 polymorphic variants in SORL1 coding region, 43 of which were considered non-synonymous and among those 17 were classified as probably or possibly damaging by Polyphen2 (Supplementary Table S4). No single-variant test provided a significant association for $\mathrm{AD}$ risk. The combined gene-based G-SKAT analysis of the 45 coding non-synonymous variants did not find any significant association with $\operatorname{LOAD}(P=0.337)$, nor did the analysis of the 21 variants considered probably damaging ( $P=0.596$; Supplementary Table S4). 


\section{DISCUSSION}

Gene-based analyses provide more power to detect association than single-variant analyses, especially when these variants present a low frequency ( $\mathrm{MAF}<1 \%$ ). This is supported by previous studies in which multiple independent variants have been reported as causative ${ }^{14}$ or increase AD risk in APP, PSEN1, PSEN2, APOE, TREM2, PLD3 and ABCA7. ${ }^{15-18}$

In this study, we have observed similar effect of rare variants in SORL1 as in previous studies, both at single-variant level (rs117260922:G > A), and at a gene-based level in the sEOAD cohort, adding support to the role of rare missense variants in SORL1 as risk factors for AD. Although our sEOAD sample size (217 cases and 169 controls) was smaller than that of Nicolas et al. ${ }^{10}$ (484 cases and 498 controls), we still had enough statistical power (83.4\%) to replicate the original finding (gene-based $\mathrm{OR}=5.03$ ).

Our results suggest that the effect size of SORL1 may be lower than originally reported. However, it is important to note that in this data set we performed genotyping and not sequencing; therefore, we may have missed additional variants that could affect our OR estimation and $P$-value.

The lack of significant findings in the sLOAD data set may possibly be due to a combination of limited power and the fact that we were only looking at exome-chip variants, not sequencing data. Instead, the lack of significant association on our fLOAD data set raises some concerns. We analyzed a very large data set containing sequencing data for familial LOAD (345 families, 1190 individuals), but we were unable to find a significant association at the gene-based level, even though we found some variants that seem to segregate in some small families. On the other hand, the different degree of association of SORL1 across the different populations (French, ${ }^{10}$ CaribbeanHispanic $^{9}$ and European American), reinforces the idea that the specific effect size for these low-frequency and rare variants are population-specific. Therefore, in order to replicate these studies, single-variant analyses are not optimal. Instead resequencing of the entire genes in well-matched populations are the most idealistic approach to determine whether these genes are really associated with disease status, and to determine the real effect size of the association.

\section{CONFLICT OF INTEREST}

The authors declare no conflict of interest.

\section{ACKNOWLEDGEMENTS}

We thank contributors who collected samples used in this study, as well as patients and their families, whose help and participation made this work possible. This work was supported by grants from the National Institutes of Health (R01-AG044546, P01-AG003991, and R01-AG035083), and the Alzheimer Association (NIRG-11-200110, BAND-14-338165 and BFG-15362540). This research was conducted while CC was a recipient of a New Investigator Award in Alzheimer's disease from the American Federation for Aging Research. CC is a recipient of a BrightFocus Foundation Alzheimer's Disease Research Grant (A2013359S). The recruitment and clinical characterization of research participants at Washington University were supported by NIH P50 AG05681, P01 AG03991, and P01 AG026276. Samples from the National Cell Repository for Alzheimer's Disease (NCRAD), which receives government support under a cooperative agreement grant (U24 AG21886) awarded by the National Institute on Aging (NIA), were used in this study. NIALOAD samples were collected under a cooperative agreement grant (U24 AG026395) awarded by the National Institute on Aging.

\section{AUTHOR CONTRIBUTIONS}

Study design was developed by CC and MVF. Phenotype data was collected by SM, JN and RC. ADRC, NIA-LAOD and AG recruited participants. Sample collection was conducted by JM, AG, JN and RC. DNA extraction was performed by DC, CB and KB. Genotyping was conducted by KB. Statistical analyses were performed by YD, BH, JA and MVF. Manuscript was drafted by MVF and CC. Funds support was received from CC. All authors read and approved the final manuscript.

1 Mattson MP: Pathways towards and away from Alzheimer's disease. Nature 2004; 430: 631-639.

2 Rogaeva E, Meng Y, Lee JH et al: The neuronal sortilin-related receptor SORL1 is genetically associated with Alzheimer disease. Nat Genet 2007; 39: 168-177.

3 Webster JA, Myers A, Pearson JV et al: SORL1 as an Alzheimer's disease predisposition gene? Neurodegener Dis 2008; 5: 60-64.

$4 \mathrm{Li} \mathrm{H}$, Wetten S, Li L et al: Candidate single-nucleotide polymorphisms from a genomewide association study of Alzheimer disease. Arch Neurol 2009; 65: 45-53.

5 Seshadri S, DeStefano A, Au R et al: Genetic correlates of brain aging on MRI and cognitive test measures: a genome-wide association and linkage analysis in the Framingham Study. BMC Med Gene 2007; 8(Suppl 1): S15.

6 Bettens K, Brouwers N, Engelborghs S et al: SORL1 is genetically associated with increased risk for late-onset Alzheimer disease in the Belgian population. Hum Mutat 2008; 29: 769-770.

7 Tan EK, Lee J, Chen CP et al: SORL1 haplotypes modulate risk of Alzheimer's disease in Chinese. Neurobiol Aging 2009; 30: 1048-1051.

8 Meng Y, Lee JH, Cheng R, George-Hyslop PS, Mayeux R, Farrer L: Association between SORL1 and Alzheimer's disease in a genome-wide study. Neuroreport 2007; 18 : 1761-1764.

9 Vardarajan BN, Zhang Y, Lee JH et al: Coding mutations in SORL1 and Alzheimer disease. Ann Neurol 2015; 77: 215-227.

10 Nicolas G, Charbonnier C, Wallon D et al: SORL1 rare variants: a major risk factor for familial early-onset Alzheimer's disease. Mol Psychiatry 2015; 21: 831-836.

11 Cingolani $P$, Platts $A$, Wang Le $L$ et al: A program for annotating and predicting the effects of single nucleotide polymorphisms, SnpEff: SNPs in the genome of Drosophila melanogaster strain w1118; iso-2; iso-3. Fly (Austin) 2012; 6: 80-92.

12 Wu MC, Seunggeum L, Tianxi C, Li Y, Boehnke M, Lin X: Rare-variant association testing for sequencing data with the sequence kernel association test. Am J Hum Genet 2011; 89: 82-93.

13 Wang X, Lee S, Zhu X, Redline S, Lin X: GEE-based SNP set association test for continuous and discrete traits in family-based association studies. Genet Epidemiol 2013; 37: 778-786.

14 Cruchaga C, Chakraverty S, Mayo $\mathrm{K}$ et al: Rare variants in APP, PSEN1 and PSEN2 increase risk for $A D$ in late-onset Alzheimer's disease families. PLoS One 2012; 7: e31039.

15 Hollingworth $\mathrm{P}$, Harold D, Sims $\mathrm{R}$ et al: Common variants at ABCA7, MS4A6A/ MS4A4E, EPHA1, CD33 and CD2AP are associated with Alzheimer's disease. Nat Genet 2011; 43: 429-435.

16 Cruchaga C, Karch CM, Jin SC et al: Rare coding variants in the phospholipase D3 gene confer risk for Alzheimer's disease. Nature 2014; 505: 550-554.

17 Jin SC, Sheng C, Benitez BA et al: Coding variants in TREM2 increase risk for Alzheimer's disease. Hum Mol Genet 2014; 23: 5838-5346.

18 Del-Aguila JL, Koboldt DC, Black K et al: Alzheimer's disease: rare variants with large effect sizes. Curr Opin Genet Dev 2015; 33: 49-55.

Supplementary Information accompanies this paper on European Journal of Human Genetics website (http://www.nature.com/ejhg) 